\title{
To triage or not to triage? The history and evidence for this model of care in psychiatry
}

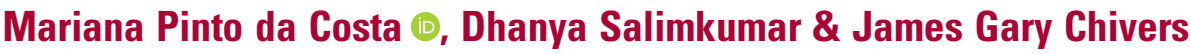

\begin{abstract}
Mariana Pinto da Costa, MD, MSc, $\mathrm{PhD}$, is a consultant psychiatrist at South London and Maudsley NHS

Foundation Trust; she is also a senior lecturer at the Institute of Psychiatry, Psychology \& Neuroscience, King's College London, UK, and at the Institute of Biomedical Sciences Abel Salazar at the University of Porto,

Portugal. She has experience working on male and female psychiatric triage wards in different NHS trusts in the London area. Dhanya Salimkumar, $\mathrm{MBChB}, \mathrm{MSc}, \mathrm{BSc}$, is a core psychiatry trainee at East London NHS Foundation Trust, UK. She is a postgraduate medical doctor and studied at the University of Warwick after having worked in the trust as a social therapist. She has experience working on a female triage ward and is currently working on a general adult ward. James Gary Chivers, BMBS, MRCPsych, is a specialist registrar in general adult psychiatry at South London and Maudsley NHS Foundation Trust, London, UK. He studied medicine at Brighton and Sussex Medical School, where he developed a passion for psychiatry and has worked in London for the past 6 years, with his most recent post being on a male triage ward at the Ladywell Unit in south-east

London.

Correspondence Dr Mariana Pinto da Costa. Email: mariana.pintodacosta@kcl.ac.uk
\end{abstract}

First received 24 Jul 2021 Final revision 28 0ct 2021 Accepted 2 Nov 2021

\section{Copyright and usage}

(C) The Author(s), 2021. Published by Cambridge University Press on behalf of the Royal College of Psychiatrists. This is an Open Access article, distributed under the terms of the Creative Commons Attribution licence (https://creativecommons. org/licenses/by/4.0/), which permits unrestricted re-use, distribution, and reproduction in any medium, provided the original work is properly cited.

\section{SUMMARY}

Triage wards were introduced as a new model of psychiatric in-patient care in 2004. However, there is limited evidence comparing them with the traditional in-patient models of care. This article reviews the history of triage wards, their principles, the evidence for this model (e.g. length of in-patient stay, readmission rates, staff and patient satisfaction) and the development of assessment wards based on the triage model of care. The evidence shows that the triage model has higher rates of rapid discharge, with a greater proportion of 'acute care' performed in the community with the support of home treatment teams. This leads to lower bed occupancy in the triage wards without increased rates of readmission or a worse patient experience of in-patient care. However, overall staff experience was better in the traditional model, given that staff satisfaction rates were lower on locality wards in settings with triage systems in place. Future research should explore the potential impact on home treatment teams, and the rates of serious incidents due to the high number of acutely unwell patients on triage wards.

\section{LEARNING OBJECTIVES}

After reading this article you will be able to:

- understand the rationale for the introduction of the triage model of care

- understand the principles of the triage model of care

- understand the evidence for and against the triage model of care.

\section{KEYWORDS}

Triage model; in-patient; locality wards; acute care; psychiatry.

The triage model of in-patient psychiatric care in the National Health Service (NHS) was introduced in south-east London in 2004, and described for the first time in the literature the following year in a paper in the Psychiatric Bulletin (Inglis 2005). This new model of care was introduced to address concerns with traditional in-patient care (Fig. 1), which at this time was described as ineffective, inefficient, and poorly organised' (Muijen 1999). The concerns about the quality of care in the traditional model comprised growing rates of patient dissatisfaction, serious untoward incidents, staff burnout and high staff turnover (Inglis 2005). High rates of bed occupancy (often greater than $100 \%$ ) in the traditional in-patient system led to problems with 'throughput' of patients, leading to subsequent undue delays in admission to in-patient wards (Greengross 2000). Despite these numerous concerns regarding traditional in-patient care, and the fact that psychiatric patients in the UK were known to have longer hospital stays, above international best standards (Sainsbury Centre for Mental Health 2002), in-patient care continued to consume the largest proportion of the mental health budget and employ the greatest number of staff (Department of Health 2002). In 2000, the NHS Plan mandated the roll-out of 335 home treatment teams (HTTs) across the UK in attempts to reduce hospital admissions or to facilitate earlier discharges, although evidence for this was mixed, with little indication of reductions in the use of in-patient beds (Jacobs 2011).

With input from Martin Baggaley, who at the time was Clinical Director at South London and Maudsley NHS Trust (SLaM), the first triage ward was opened in the grounds of the University Hospital Lewisham, at the Ladywell Unit, in 2004. Prior to the operation of the triage model, the Ladywell mental health unit had housed 70 acute admission beds split between three wards in a traditional in-patient model. In this system, there were also an additional eight low-intensity beds within a pre-discharge unit.

The opening of the new triage system involved a 'whole system approach' including restructuring of the wards at the Ladywell Unit. Under the new system, the number of beds on the three 'locality wards' at this site was reduced from 23 to 18 , and the new 16-bed, mixed-gender triage unit was opened. The eight low-intensity (pre-discharge) beds were lost, but a total of 70 acute beds were available at this site in the new system structure (Inglis 2005). 


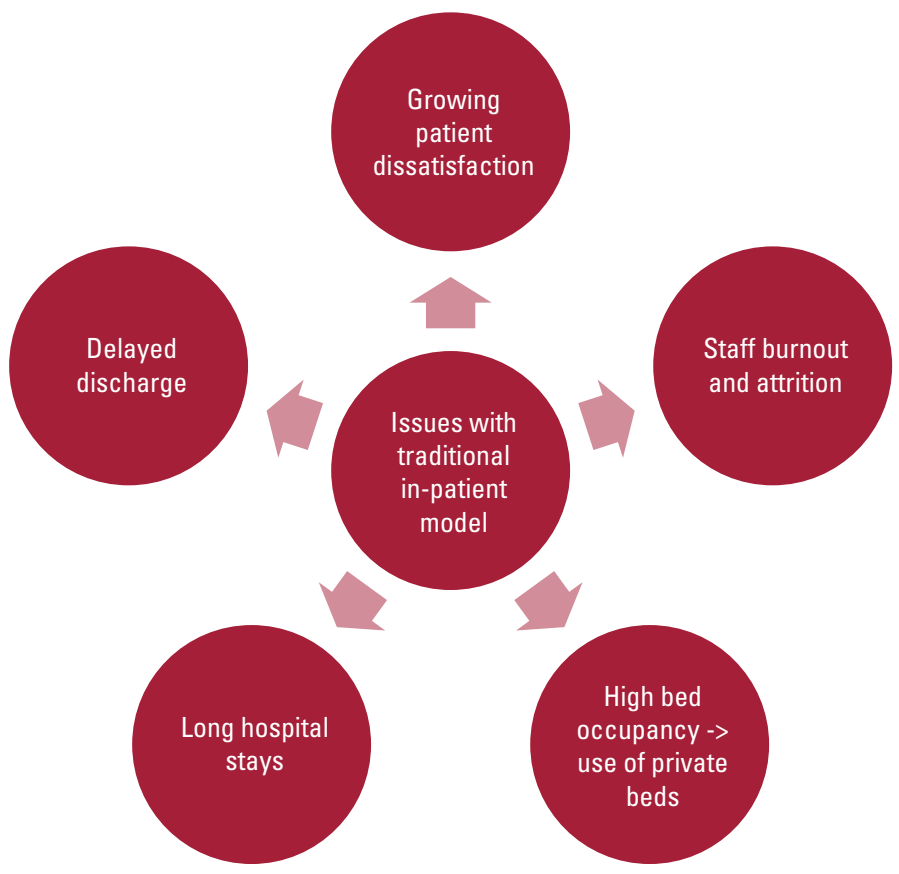

Problems identified with the traditional in-patient model of psychiatric care in England (Muijen 1999).

\section{Principles of the triage model of care}

The term 'triage' illustrates that patients were assessed and prioritised according to need (Inglis 2005). All in-patient admissions from the Lewisham area were admitted to the triage ward for assessment of the most appropriate intervention. Following an assessment period, patients were either discharged to primary care, a community mental health team (CMHT) or home treatment team (HTT), or admitted to one of the three locality wards (Fig. 2). The length of stay in the triage ward was limited to a maximum of 7 days, following which the patient was transferred to their locality ward.

The rationale was that a concentrated assessment phase on the triage ward, including consultant input 6 days a week, would result in decisions and care being delivered in a more timely and efficient fashion. It was highlighted that funding for the consultant was freed up from no longer having to admit patients to private in-patient units. In the traditional model of in-patient care, decisions on treatment or discharge were often delayed and made on weekly ward rounds (Inglis 2005). The aim in the triage model was to initiate management plans early on, with the goal of reducing the length of in-patient stay and preventing delayed discharge. The rates of bed occupancy were more optimal (approximately $70 \%$ ), meaning that beds were more readily available to patients needing to be admitted from the community or emergency departments. The aim was also to be able to invest in care delivery the time that would have been spent looking for beds. The core principles of the original triage model implemented in Lewisham are illustrated in Fig. 3.

\section{'Focused purposeful admissions'}

There was a daily multidisciplinary team (MDT) discussion of both current and planned admissions, allowing plans to be drawn up and more collateral information to be sought for planned admissions if required.

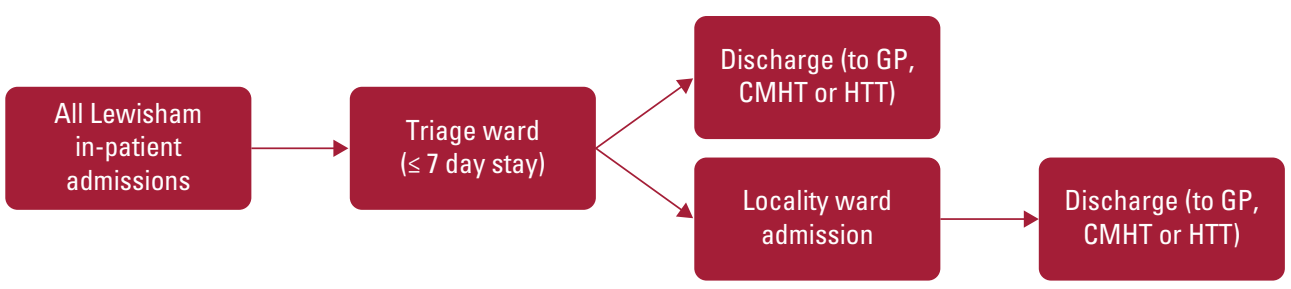

The journey through the psychiatric triage model from admission to discharge (Inglis 2005). GP, general practitioner; CMHT, community mental health team; HTT, home treatment team. 

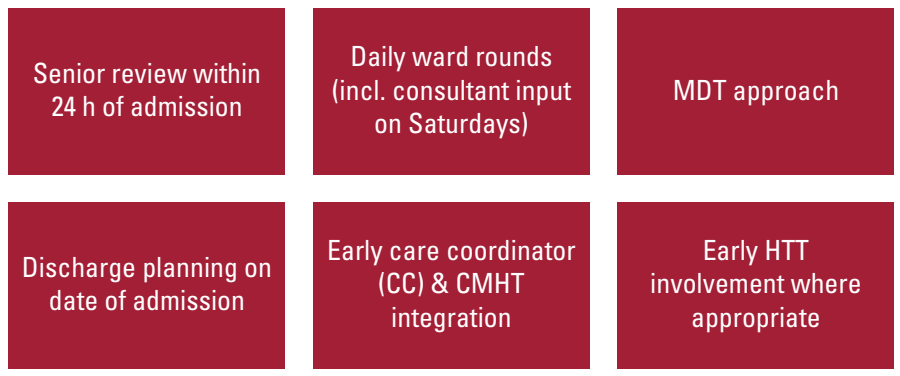

Early HTT

involvement where

appropriate

Core principles of the psychiatric triage model (Inglis 2005). MDT, multidisciplinary team; HTT, home treatment team; CMHT, community mental health team.

On admission to the ward, a comprehensive nursing and medical assessment took place, as well as a review by a senior psychiatrist. Discharge planning began on the day of admission, with involvement of CMHTs or HTTs as soon as possible. Care coordinators were involved in the assessments and in discharge planning where possible. The importance of involving both community teams and locality ward teams in assessments was stressed, with locality consultants being able to visit the triage ward to contribute to assessments (Inglis 2005).

\section{Medical staffing and early senior review}

The ward was staffed with the equivalent of one fulltime consultant (two part-time consultants), a specialist registrar $(\mathrm{SpR})$ and a senior house officer (SHO). The SpR and SHO were solely employed on the triage ward and had no other clinical duties. The consultant was present at the daily MDT review, where new patients were highlighted as well as patients requiring further medical input. There was consultant input on the ward 6 days a week (including Saturdays), with the aim that each new admission had senior medical input within 24 h (Inglis 2005).

\section{MDT approach}

Multidisciplinary working was an integral part of the triage model. Each patient was discussed by the MDT on the ward daily, with these discussions informing care plan and treatment decisions. There was input from social workers daily, meaning that any issues with benefits, care packages and housing could be addressed early on in the admission. A dual diagnosis nurse consultant was also available to provide input and advice relating to patients with substance misuse disorders (Inglis 2005).

\section{Information technology}

Patient information from the electronic notes was displayed on a large screen during the daily reviews. This enabled the entire MDT to review details, including recent contact with CMHTs, care programme approach (CPA) meetings, discharge summaries, risk assessments and other useful information.

A 'running entry' was made in the notes during ward rounds, serving as a useful summary of each patient's progress on the triage ward and ultimately forming the basis for the discharge summary. There was frequent use of email contact to clarify details and advice from community teams and other parties involved in the patients' care. The team found that responses to emails from community and other teams were often received while ward rounds were still in progress, meaning that decisions could be made without delay (Inglis 2005).

\section{Ward environment}

The triage ward at the Ladywell Unit was mixed gender, but with male and female beds in separate areas. Staff had been involved in the refurbishment and design of the ward, including input on the furnishings and fittings. There was an emphasis on providing a comfortable and safe environment conducive to good-quality assessments (Inglis 2005).

\section{Early results}

In the first 6 months of the triage care in Lewisham, there were 406 in-patient admissions: 170 were discharged home within 7 days, 37 were transferred to the borough responsible for their care and 199 were admitted to a Lewisham locality ward (Inglis 2005) (Fig. 4).

The average bed occupancy on the triage unit was $70 \%$, meaning that patients could be admitted immediately when needed. Some beds were also provided for the three other boroughs within SLaM when their in-patient systems were unable to accommodate new patients at the time of admission.

The high rates of rapid discharge, along with the bed occupancy rates, suggested that the triage system had brought major improvements to inpatient care (Inglis 2005). It was also postulated 


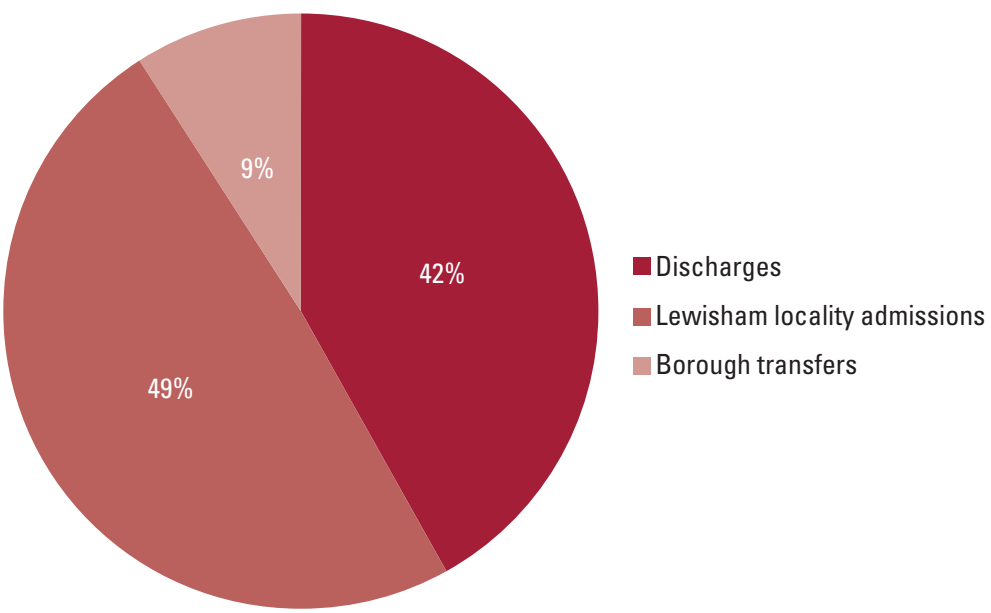

In-patient admission figures for the initial 6 months of the psychiatric triage ward in Lewisham, London (Inglis 2005).

that this would be of benefit to patients on locality wards, given the staff time freed up from not having to search for beds or respond to unexpected or poorly planned admissions. It was suggested that this time could then be spent on therapeutic engagement and on delivering care to the locality ward patients (Inglis 2005).

\section{More questions than answers?}

Despite the positive early figures, questions were raised about the number of adverse incidents on the ward, rates of 1:1 nursing observations and levels of sickness among nursing staff (Inglis 2005). Hayes et al (2012) reviewed data from admissions on an acute assessment ward in north London and found no significant differences in incidents of verbal or physical aggression in comparison with data gathered from acute psychiatric wards in the City 128 Study (Bowers 2007) but did find that the incidence of patients' self-harm was significantly lower on the assessment ward $(P=0.001)$.

Figure 5 illustrates various questions about the triage model.

\section{Is triage care more efficacious?}

In 2014 the triage model was expanded within SLaM to include three out of their four boroughs: Lewisham, Croydon and Lambeth (Debelle 2017). At this time, the concerns about in-patient psychiatric care in the UK persisted, with ongoing pressures to search for efficiency and explore ways of avoiding or shortening hospital admissions and reducing associated costs in healthcare systems. Although London had the lowest median length of stay for the UK (38 days), this continued to be higher than the average for countries in the Organisation for Economic Co-operation and Development (21 days for an affective disorder and
38 days for psychosis), and average bed occupancy rates were $85-100 \%$ or higher (Sainsbury Centre for Mental Health 2005; Williams 2014).

The efficacy and cost-effectiveness of the triage model at reducing the length of in-patient stay and readmission rates was reported in 2014, where the triage model was compared with the traditional inpatient model (Williams 2014). The records of 1834 hospital admissions were examined in 2009 over a 1-year period, within two comparable innercity systems in a large mental health trust (935 admissions in the triage system and 899 admissions in the traditional system). There were no statistically significant differences in the characteristics of patients across the two systems. There was a slightly higher proportion of out-of-area patients admitted in the triage system $(24 \%$, versus $21 \%$ in the traditional system). This study demonstrated that more admissions to the triage system resulted in 'rapid discharge' (less than 10 days' in-patient stay) from hospital: 403 rapid discharges (43\% of admissions), versus 259 rapid discharges (29\% of admissions) in the traditional in-patient system (Fig 6). The odds of rapid discharge for catchment area patients were over twice as high in the triage system compared with the traditional model $(P<0.001)$. There was no statistically significant difference in the odds of rapid discharge for out-of-area admissions. For each patient, a 1-year follow-up period post-discharge was investigated to ascertain any potential effects on readmission rates. The 1 -year readmission rate across both systems was $35 \%$, with no significant difference between systems.

\section{Does the triage model reduce length of hospital stay?}

Looking at the 'administrative length of stay', although there appeared to be shorter mean (45 v. 


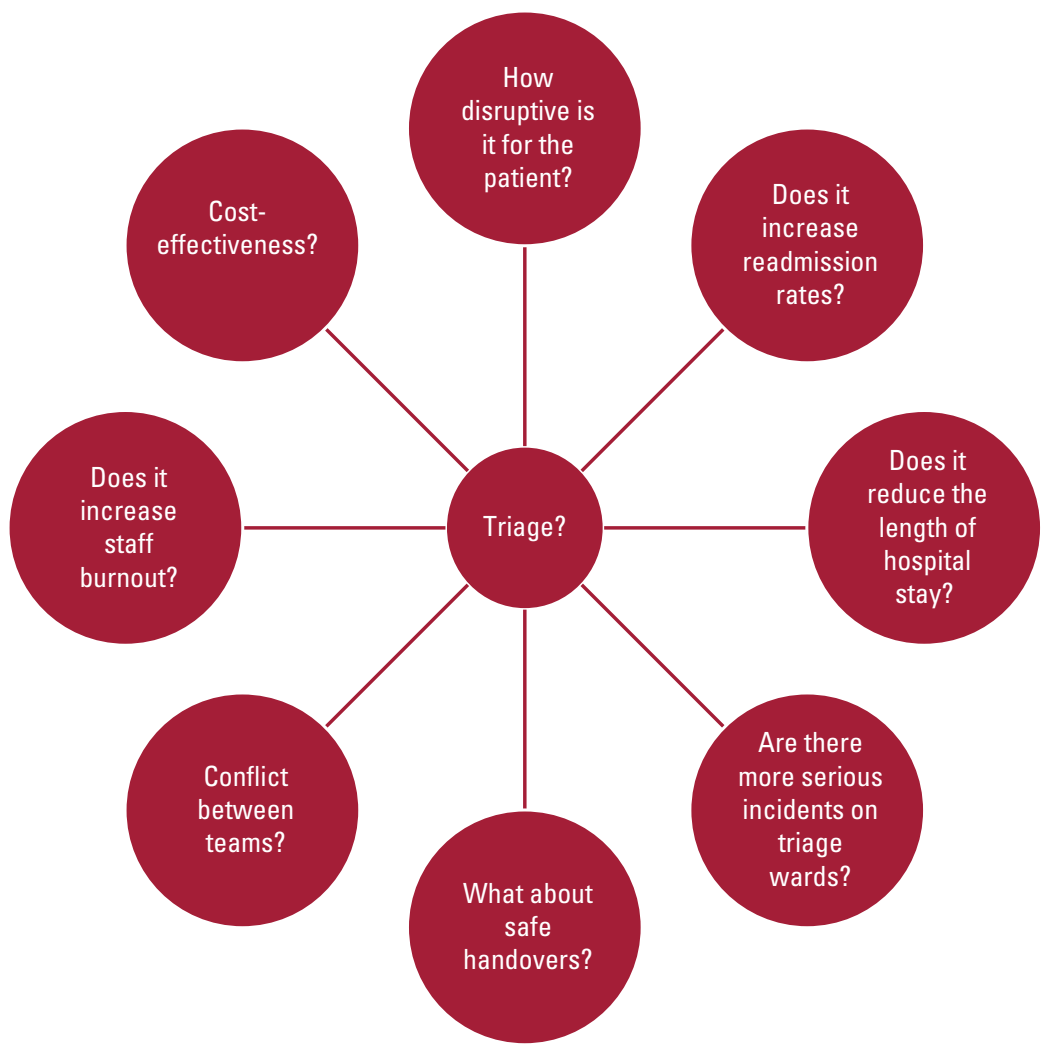

FIG 5

Questions about the psychiatric triage model (Inglis 2005).

47 days) and median (16 v. 22 days) in-patient stays in the triage model, this was not statistically significant (for the means $P=0.364$ ). Considering beddays, this suggested that there had been more use of leave periods in the triage system. When this was accounted for by excluding leave days from the measure of the length of stay, the mean number of bed-days in the triage system was lower by over 8 days $(P=0.030)$ (Williams 2014). However, there was a notable and significant

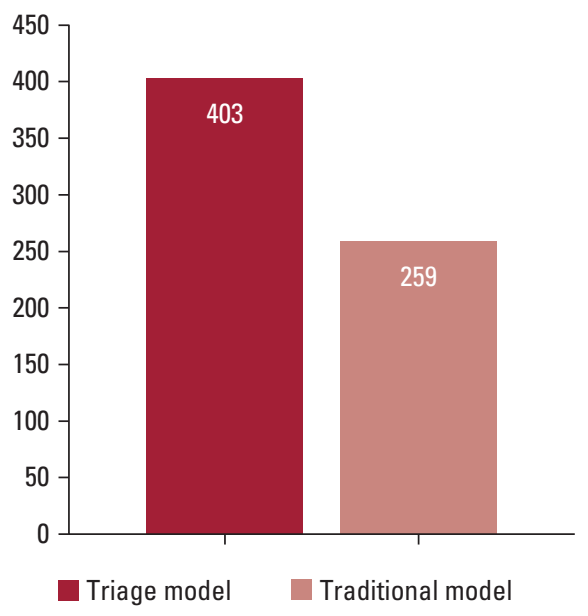

Total number of rapid discharges for the psychiatric triage and traditional care models (Williams 2014). difference in the use of HTTs across systems, with the triage model utilising HTTs for longer periods and more frequently (in 32 v. 26\% of admissions, $P=0.006)$. When the additional days spent with HTTs were accounted for, by adding them to inpatient days to calculate 'duration of acute care', the mean duration of acute care was longer in the triage system (Fig. 7), although this was not statistically significant $(P=0.076)$. Crossley \& Sweeney (2020) conducted a retrospective case-cohort study investigating patient and service-level factors affecting the length of in-patient stay across wards in an inner-city mental health trust in Manchester. The results of their study showed that admission to and discharge from the short-stay assessment ward were associated with a significantly reduced overall length of in-patient stay $(P<0.001)$ and did not significantly prolong length of stay for those who subsequently required admission to an acute ward.

\section{Which system is more cost-effective?}

A cost analysis of both systems was conducted by obtaining figures for the cost of HTT days and inpatient days and using these to calculate the mean costs of 'acute care episodes' (HTT days + inpatient days). Each system incurred costs of around $£ 15000$ for an average acute care episode. 


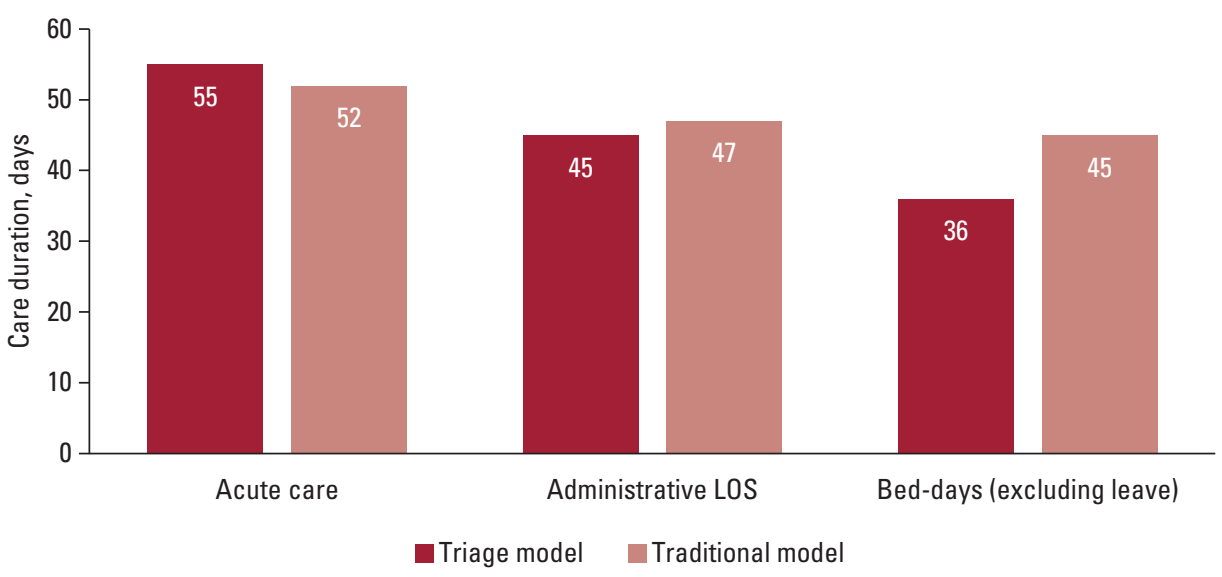

Duration of care for the psychiatric triage and traditional care models (Williams 2014). LOS, length of stay.

There were no statistically significant differences in the cost analyses (Williams 2014).

\section{What about patient and staff experience?}

Since the failures in patient care at Mid Staffordshire NHS Foundation Trust were reported in the independent inquiry by Sir Robert Francis QC, it has been emphasised that patient experience should be of central importance in healthcare delivery (Francis 2013). Csipke et al (2016) assessed patient and staff perceptions within the triage and traditional systems over an 18-month period using self-report questionnaires, with $61 \%$ of eligible patients $(n=$ $454)$ and $57 \%$ of eligible staff $(n=284)$ taking part.

\section{Patient experience}

There were no significant differences found between patients' perception or satisfaction across both models of care, although concerningly, patient satisfaction decreased in both models over the period of the study (Csipke 2016). This study measured 'patient-perceived meaningful contacts' with health professionals across both models. Although both systems had similar numbers of nursing staff, patients in the triage system reported approximately half the number of contacts with nursing staff compared with those receiving traditional care (this was not including contacts with care coordinators). Notably, patients in the triage wards were significantly less likely to take part in activities or to have contact with other professionals.

\section{Staff experience}

The most significant trend was a decline in staff perception of in-patient care in both systems over time (Csipke 2016). There were no significant differences between triage wards, locality wards in the triage system or traditional in-patient wards. Staff burnout was assessed using the Maslach Burnout Inventory and there were no significant differences in baseline rates of burnout across the two models of care. However, worsening rates of burnout over time were observed in the traditional model, whereas burnout rates in the triage system remained relatively stable. Burnout was lowest on locality wards in the triage system, although conversely, staff satisfaction on these locality wards was actually much lower. This accounted for a significant difference in staff experience across the two models, with the traditional model faring better in terms of staff satisfaction.

Reasons behind this difference in staff experience are clearly not explained by levels of burnout. The finding that locality wards in the triage system had the lowest rates of both staff satisfaction and burnout (Csipke 2016) may be explained by the higher numbers of delayed discharges on these wards and the delays in waiting for social care or accommodation problems to be addressed.

\section{Change of nomenclature: the assessment model}

In other parts of England, there has been a change in nomenclature, from triage wards to assessment wards or units, with the development of new services based on the assessment model of care; particularly, in recent years, we have seen the development of psychiatric decision units (PDUs) in England. PDUs are $24 \mathrm{~h}$ mental health acute assessment units with the ability to assess informal patients for up to $72 \mathrm{~h}$, drawing from features of a triage ward as regards enhanced assessment on admission to ascertain whether admission to an acute psychiatric ward is required. A national survey conducted by Goldsmith et al (2021) reported that there were six PDUs across England but there was significant heterogeneity between their characteristics, including differences in the aim of this service and referral pathways. Trethewey et al (2019) evaluated the impact of a PDU in Birmingham and Solihull 
Mental Health Foundation Trust on in-patient admissions and found a $26 \%$ reduction in inpatient psychiatric admissions from the local emergency department.

\section{To triage or not to triage}

Existing research into triage wards has shown high rates of rapid discharge, improved bed occupancy and lower number of days spent in wards within the triage system. Indeed, by 2014, South London and Maudsley NHS Foundation Trust had expanded the triage system to cover three out of its four south London boroughs (Debelle 2017). Despite this, by 2018 this had reduced to just one borough (Lewisham) operating the triage system. The reasons for dismantling the triage wards were not disclosed but the triage model was implemented in various other trusts across England (Goldsmith 2021).

After reviewing the evidence on this subject, it is clear that various unknowns remain. Wykes et al (2018) found that in the triage model of care, a greater proportion of patients were followed up by HTTs in comparison to those admitted under the routine care system (32\% and $26 \%$ respectively; $P=0.006)$. It would be useful to explore the effect this has on HTTs as regards case-loads, staff satisfaction and staff retention. It is not clear whether the triage system confers the same benefits outside of busy inner cities where bed occupancy rates can differ. There is also not enough evidence on whether the level of acuity on triage wards - with patients with significable, unpredictable needs - could contribute to higher rates of adverse incidents. Importantly, it seems that multiple other factors play a role in in-patient care and that a shared responsibility for improvement lies also with parties outside of the healthcare system. In particular, one would expect that making appropriate accommodation or social support more readily accessible could have a significant impact on overall length of stay by reducing delayed discharges.

The Inglis, Williams and Csipke articles (Inglis 2005; Williams 2014; Csipke 2016) suggest that at the very least, the triage model is not inferior to the traditional in-patient system. Moreover, improved rates of bed occupancy in the triage system should not be understated, particularly as they would be expected to lead to more rapid transfer of acutely unwell patients from emergency departments to mental health in-patient units. This should reduce the need for trusts to outsource acute care to private hospitals, which can vary significantly in terms of their proximity to the patient's catchment area and also in the quality of care offered. Attempting to contain acutely disturbed patients in busy emergency departments while they await an in-patient bed is challenging and not uncommon (The Strategy Unit 2019). It is known that 'boarding' of patients in emergency departments has been shown to lead to crowding, poor patient experience, lower quality of care, delays in treatment, increased morbidity and mortality, and lost revenue (Pearlmutter 2017).

\section{Conclusions}

The triage system is a valuable model that works at least as well as the traditional model, but with added benefits of: increasing rapid discharges; the

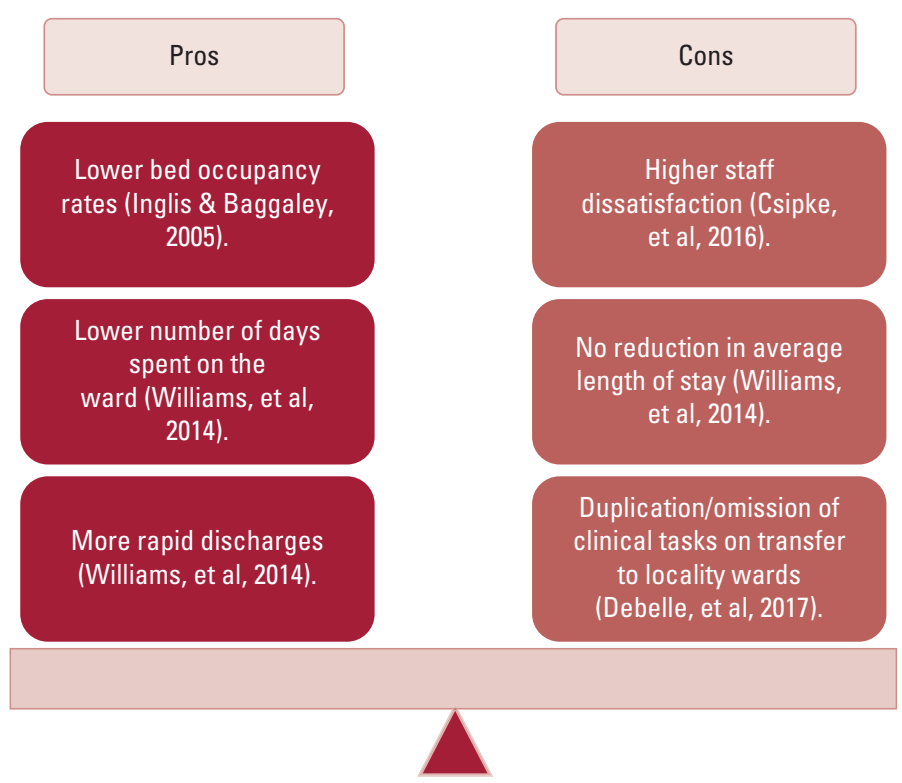


increased presence of social workers and dual-diagnosis professionals; decreasing the number of days patients spend in the ward; improving bed occupancy and throughput of patients; and reducing the need to outsource in-patient care to private providers (Fig. 8), without negative effects on 1-year readmission rates. The benefits are likely due to the increased presence of senior doctors and MDT professionals, including those from social care and substance misuse teams, to help facilitate quicker discharge. However, readmission rates and average lengths of stay do not differ across the two systems. It also appears that staff satisfaction is lower within locality wards where there is a triage model, with studies suggesting this could be due to frustrations with the slower patient turnover and delayed discharges but no data have been collected to verify this.

The triage model is a relatively understudied system, and it is unclear how staff on home treatment teams might be affected or whether there is much difference in the rates of serious incidents on triage wards. Despite the recent dismantling of triage wards in SLaM, the triage model has been implemented in various other trusts across England. However, there is not enough information on the existing triage and assessment wards to see whether there are fundamental differences or adaptions of the triage model of care first implemented in SLaM that has led to the continued use of triage wards in these trusts. Further research into this area would help to identify what contributes to an efficient triage model of care to help alleviate pressures on mental health in-patient beds.

\section{Data availability}

Data availability is not applicable to this article as no new data were created or analysed in this study.

\section{Author contributions}

All authors contributed to writing this manuscript.

\section{Funding}

This research received no grant or funding.

\section{Declaration of interest}

M.P.C. is a member of the BJPsych Advances editorial board and did not take any part in the review or decision-making process of this manuscript.

\section{References}

Bowers L, Whittington R, Nolan P (2007) The City 128 Study of Observation and Outcomes on Acute Psychiatric Wards. National Co-ordinating Centre for the National Institute for Health Research Service Delivery and Organisation Programme (NCCSDO).

Crossley N, Sweeney B (2020) Patient and service-level factors affecting length of inpatient stay in an acute mental health service: a retrospective case cohort study. BMC Psychiatry, 20: 438

Csipke E, Williams P, Rose D, et al (2016) Following the Francis report: investigating patient experience of mental health in-patient care. British Journal of Psychiatry, 209: 35-39.

Debelle A, Dashwood M, Bird L, et al (2017) Improving handover between triage and locality wards in a large mental health trust. BMJ Open Quality, 6(e000023)

Department of Health (2002) Acute Inpatient Provision Care Provision: Mental Health Policy Implementation Guide. Department of Health.

Francis R (2013) Report of the Mid Staffordshire NHS Foundation Trust Public Inquiry. TSO (The Stationery Office)

Goldsmith LP, Anderson K, Clarke G, et al (2021) The psychiatric decision unit as an emerging model in mental health crisis care: a national survey in England. International Journal of Mental Health Nursing, 30, $955-62$

Greengross P, Hollander D, Stanton R (2000) Pressure on adult psychiatric beds. results of a national questionnaire survey. Psychiatric Bulletin, 24: $54-6$

Hayes J, Gibbons R, Outim F, et al (2012) A new model for quality improvement in acute inpatient psychiatry: observational data from an acute assessment unit. Journal of the Royal Society of Medicine short reports, 3(9): 65

Inglis G, Baggaley M (2005) Triage in mental health - a new model for acute in-patient psychiatry. Psychiatric Bulletin, 29: 255-258.

Jacobs R, Barrenho E (2011) Impact of crisis resolution and home treatment teams on psychiatric admissions in England. British Journal of Psychiatry, 199: 71-6.

Muijen M (1999) Acute hospital care: ineffective, inefficient and poorly organised. Psychiatric Bulletin, 23: 257-9.

Pearlmutter MD, Dwyer KH, Burke LG, et al (2017) Analysis of emergency department length of stay for mental health patients at ten Massachusetts emergency departments. Annals of Emergency Medicine, 70(2): 193-202.e16.

Sainsbury Centre for Mental Health (2002) An Executive Briefing on Adult Acute Inpatient Care for People with Mental Health Problems. Sainsbury Centre for Mental Health.

Sainsbury Centre for Mental Health (2005) Acute Care 2004: A National Survey of Adult Psychiatric Wards in England. Sainsbury Centre for Mental Health.

The Strategy Unit (2019) Exploring Mental Health Inpatient Capacity. The Strategy Unit.

Trethewey SP, Deepak S, Saad S, et al (2019) Evaluation of the Psychiatric Decisions Unit (PDU): effect on emergency department presentations and psychiatric inpatient admissions. Postgraduate Medical Journal, 95(1119): 6-11.

Williams P, Csipke E, Rose D, et al (2014) Efficacy of triage system to reduce length of hospital stay. British Journal of Psychiatry, 204: 480485

Wykes T, Csipke E, Rose D, et al (2018) Patient involvement in improving the evidence base on mental health inpatient care: the PERCEIVE programme. Programme Grants for Applied Research, 6(7). 


\section{MCQs:}

Select the single best answer for each question

1 Multiple problems with the traditional inpatient model of care have been highlighted. Which one of the following was not a concern regarding the traditional in-patient model?

a high rates of staff burnout

b bed occupancy rates of $>100 \%$

c growing patient dissatisfaction

d high utilisation of home treatment teams in discharge plans

e average lengths of stay longer than international best standards.
2 Which of the following is not a principle of triage ward care?

a length of stay on triage ward is limited to 14 days

b consultant input on Saturdays

c senior review within $24 \mathrm{~h}$ of admission

d early home treatment team involvement where appropriate

e discharge planning from the day of admission.

3 Inglis \& Baggaley published figures from the first 6 months of operating the triage model in Lewisham. What was the average bed occupancy rate within the triage system?

a $60 \%$

b $90 \%$

c $80 \%$

d $70 \%$

e $>100 \%$.
4 Across both the triage and traditional systems, the readmission rate within 1 year in Williams et al's (2014) study was:

a $35 \%$

b $45 \%$

c $55 \%$

d $20 \%$

e $10 \%$

5 As regards the experience across both the triage and traditional in-patient models:

a burnout is higher on triage wards

b patient experience is significantly better in the traditional model

c there is more staff dissatisfaction reported on locality wards within the triage system

d burnout is higher on locality wards

e patient experience is significantly better on triage wards. 